\title{
Atomistic Simulation of Ceramic/Metal Interfaces: $\{222\} \mathrm{MgO} / \mathrm{Cu}$
}

\author{
R. Benedek \\ D.N. Seidman \\ L.H. Yang \\ This paper was prepared for submittal to the
Atomic Structure and Chemistry on Boundaries/Interfaces
Tempe, $A Z$ \\ January 8-11, 1997
}

February 1997

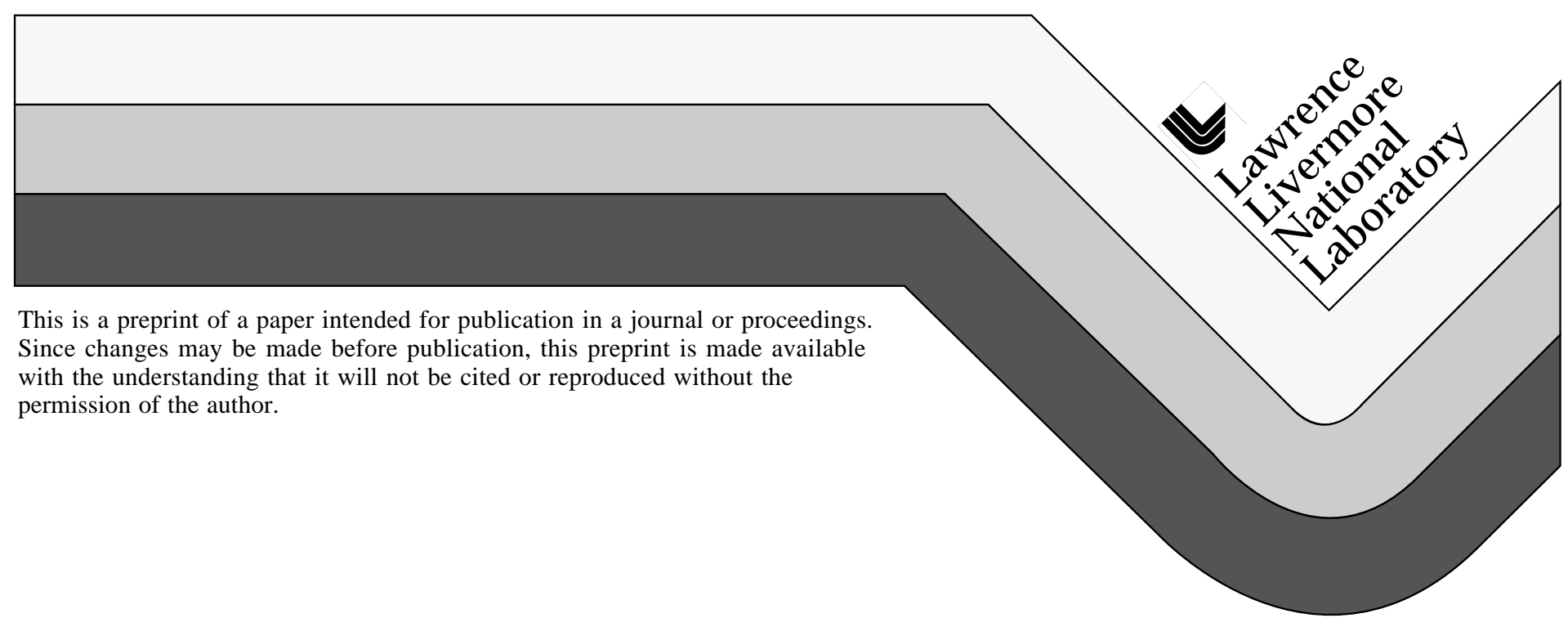




\section{DISCLAIMER}

This document was prepared as an account of work sponsored by an agency of the United States Government. Neither the United States Government nor the University of California nor any of their employees, makes any warranty, express or implied, or assumes any legal liability or responsibility for the accuracy, completeness, or usefulness of any information, apparatus, product, or process

disclosed, or represents that its use would not infringe privately owned rights. Reference herein to any specific commercial product, process, or service by trade name, trademark, manufacturer, or otherwise, does not necessarily constitute or imply its endorsement, recommendation, or favoring by the United States Government or the University of California. The views and opinions of authors expressed herein do not necessarily state or reflect those of the United States Government or the University of California, and shall not be used for advertising or product endorsement purposes. 
Atomistic Simulation of Ceramic/Metal Interfaces: $\{222\} \mathrm{MgO} / \mathrm{Cu}$

\author{
R. Benedek and D. N. Seidman
}

Materials Science and Engineering Department and Materials Research Center, Northwestern University, Evanston, IL 60208-3108

\author{
L. H. Yang \\ Condensed Matter Physics Division, Lawrence Livermore National Laboratory, University \\ of California, Livermore, CA 94551
}

\begin{abstract}
Atomistic simulations are performed for the $\{222\} \mathrm{MgO} / \mathrm{Cu}$ interface by local density functional theory (LDFT) methods, within the planewave-pseudopotential representation, and by molecular dynamics and statics. The electronic spectra obtained with LDFT calculations show a localized interface state within the bulk $\mathrm{MgO}$ gap, at $0.5 \mathrm{eV}$ below the Fermi energy. Adhesive energy calculations, as a function of interface spacing and translations parallel to the interface, are employed to devise an interatomic potential suitable for large-scale atomistic simulation. The interface structure, obtained with molecular dynamics (and statics) calculations based on the resultant potential, exhibits a misfit dislocation network with trigonal symmetry, and no standoff dislocations.
\end{abstract}

\title{
I. INTRODUCTION
}

Atomistic simulations are essential to fully interpret state-of-the-art atomic resolution probes (atom-probe field-ion microscopy, high resolution and Z-contrast transmission electron microscopies, electron energy loss spectroscopy (EELS), etc.) that have recently been applied to ceramic-metal interfaces. Owing to the lack of accurate yet tractable 
interface interatomic-potential models, however, little progress has been made on simulating the atomic structure of ceramic-metal interfaces (Finnis 1996) and particularly the consequences of misfit. In this work, an approach that combines local-density functional theory (LDFT) calculations with classical simulations is applied to a model ceramic/metal interface, $\{222\} \mathrm{MgO} / \mathrm{Cu}$, a system discussed in the presentation of Seidman and coworkers (Seidman et al. 1997) at the Arizona State University Workshop on Boundaries and Interfaces. LDFT calculations (Benedek et al. 1996), based on the coherent interface approximation, suggest that the most strongly bonded $\mathrm{MgO} / \mathrm{Cu}$ interface is the oxygen-terminated $\{222\}$ orientation, a result consistent with atom-probe field-ion microscope observations (Jang et al. 1993, Shashkov 1997, Shashkov et al. 1997), in which the facets of $\mathrm{MgO}$ precipitates in internally oxidized $\mathrm{Cu}$ are found to be exclusively of this type. The relatively large misfit of $\{222\} \mathrm{MgO} / \mathrm{Cu}(\approx 15 \%)$ must be accounted for in a realistic treatment of atomic structure, however, it cannot presently be included directly in the LDFT calculations, which scale rapidly with the size of the computational unit cell. Our strategy is to employ LDFT calculations to provide guidance as to an appropriate form of interatomic potential, suitable for classical simulation. Applying this potential in molecular statics calculations, we predict a misfit dislocation network of trigonal symmetry.

\section{SIMULATIONS FOR CERAMIC-METAL INTERFACES}

Classical (molecular dynamics and Monte Carlo) simulations typically proceed from a potential parametrized in terms of the atomic coordinates. To simulate ceramic/metal interfaces it is convenient (Finnis 1992) to consider total-energy formulations for which 


$$
E=E_{m}+E_{c}+E_{i},
$$

where $E_{m}$ involves only metal coordinates, $E_{c}$ involves only ceramic atom coordinates, and $E_{i}$ includes both ceramic and metal coordinates at the interface. Such formulations, if feasible, allow us to exploit existing representations of $E_{m}$ (such as the Embedded-Atom Method) and $E_{c}$ (such as the Born or Shell models) The image-charge treatments of Duffy et al. (1995), and Finnis (1992), can be cast in this form, as can the LDFT-based model proposed in this paper.

Recent calculations by Purton et al. (1996) for (nonpolar) \{002\}MgO/Ag interfaces based on the Discrete Classical Model of Finnis (1992) predicted that the lowest-energy atomic configuration corresponds to Ag over $\mathrm{O}$, in agreement with ab initio LDFT calculations. A technical difficulty (M. W. Finnis, private communication) that arises in extending the DCM to polar interfaces, such as $\{222\}$, is the ambiguity of the effective charge of ions in the terminating $\{222\}$ plane, which differs from the bulk values (nominally $2+$ and 2 - for $\mathrm{O}$ and $\mathrm{Mg}$ ); this issue does not arise, e.g., for the neutral nonpolar $\{002\}$ planes. A more general concern is the inability of image-charge formulations to treat the detailed electronic states at the interface (although Finnis (1992) notes that such formulations should not be viewed as strictly classical, but are correct to low perturbative order in a quantum mechanical treatment). A goal of the present work is to devise a formulation of $E_{i}$ that is consistent with LDFT total energy calculations for a wide variety of atomic configurations.

\section{INTERFACE POTENTIAL DERIVED FROM LDFT CALCULATIONS}


A familiar property related to interface bonding is the adhesive energy curve (Smith et al. 1994), basically the total energy vs. interface spacing. The adhesive energy curve has been calculated by ab initio LDFT for a number of different (mostly nonpolar) interfaces of $\mathrm{MgO}$ or corundum with various metals. In general, the results follow reasonably closely the so-called universal binding energy curve (Smith et al. 1994)

$$
E_{u}=-\varepsilon\left(1+z^{*}\right) \exp \left(-z^{*}\right)
$$

where the prefactor $\varepsilon$ is a specific interface bonding strength (which in our case we normalize per interface $\mathrm{Cu}$ atom), and $z^{*} \equiv\left(z-z_{0}\right) / l$ is a reduced length, expressed in terms of the equilibrium interface separation, $z_{0}$, and a scaling length, $l$. We note that all existing adhesive energy curve calculations approximate the interface as coherent, and thus neglect the misfit that exists for all real ceramic/metal interfaces. We return to that issue below.

To gain insight into interface bonding for $\{222\} \mathrm{MgO} / \mathrm{Cu}$, we have calculated a family of adhesive energy curves, corresponding to different parallel translations of the coherent $\mathrm{Cu}$ interface layer, relative to the oxygen interface layer. The calculations are based on the plane-wave-pseudopotential representation of LDFT, details of which are described elsewhere (Benedek et al. 1996). The most favorable registry for a coherent $\{222\} \mathrm{MgO} / \mathrm{Cu}$ interfaces occurs for interface $\mathrm{Cu}$ atoms equidistant from three interface $\mathrm{O}$ atoms ( the "hollow" position). The adhesive energy curve for this configuration is well described by $E_{u}$ with an equilibrium interface separation of $z_{0} \sim 1.26 \AA, \varepsilon=2.75 \mathrm{eV}$, and 
$l=0.625 \AA$. (A fit was also obtained for the Mg termination, for which of $z_{0} \sim 2.09 \AA$, $\varepsilon=1.66 \mathrm{eV}$, and $l=0.86 \AA$. This may not be physically relevant since the $\mathrm{Mg}$ termination has not been observed experimentally). For small parallel displacements from the hollow position, the adhesive energy curves deviate only slightly from the one previously determined. When the $\mathrm{Cu}$ atoms lie close to the "on top" positions directly above the oxygen atoms, however, the energy is considerably higher than the equilibrium curve for interface spacings in the vicinity of $z_{0}$. To summarize, except for configurations close to the on-top positions, the adhesive energy depends essentially only on the interface separation, and not on translations parallel to the interface. It turns out that this behavior can be fitted very well in terms of a superposition of one and two-body analytical potentials,

$$
E_{i}=E_{1}+E_{2}
$$

The one body potential

$$
E_{1}=\sum_{z_{i}} E_{u}\left(z_{i}\right)
$$

is the sum of contributions from $\mathrm{Cu}$ atoms at a distance $z_{i}$ from the ceramic interface layer, which, in our initial treatment, is assumed perfect and flat.

The two-body potential is a Born-Mayer repulsive interaction 


$$
E_{2}=A \sum_{i, j} \exp \left(-b r_{i j}\right)
$$

between pairs of $\mathrm{Cu}$ and oxygen atoms with separation $r_{i j}$ at the interface. For the $\mathrm{O}$

termination, we obtain $A=4254 \mathrm{eV}$ and $b=4.73 \AA^{-1}$. Equations (3), (4) and (5) represent the basic potential form used in our numerical simulations of interface structure.

Before proceeding, we comment briefly on the viewpoint and the principle approximations underlying the proposed model, and then generalize it to enable a more realistic treatment of misfit, which is large $(\approx 15 \%)$ for the system under consideration. The proposed shortrange interaction model, Eq.(1), is clearly a minimalist treatment, and may fail to describe finer details of the "true" potential-energy surface. Nevertheless, it is our expectation and hope that the model will reproduce the most significant structural features of the interface.

Let us review some of the key approximations. As we noted, the ceramic interface layer is treated as flat and defect free. The flatness assumption, made in the same spirit as in the schematic treatment of Vitek et al. (1995), is justified by the stiffer elastic moduli in the ceramic than in the metal. We expect to relax the flatness assumption in future work, after performing LDFT calculations to ascertain how best to parametrize the potential in the presence of modest buckling of the ceramic interface layer. One would also, in principle, like to study the effect of $\mathrm{O}$ vacancies at and near the interface, however, it is not clear whether this can be accomplished by a generalization of Eq. (1). A further approximation is the local density approximation of LDFT, which may lead, e.g., to overestimation of the prefactor $\varepsilon$ in Eq.(2). Cancellation of errors, however, is expected to reduce the error in the fitted model potential. Another approximation is the neglect of the distinction 
between fcc and hcp stacking at the interface. The LDFT adhesive energy curves described above were performed based on fcc stacking. Preliminary results indicate that the energy differences between fcc and hcp are small, of the order of $20 \mathrm{meV} /$ interface $\mathrm{Cu}$ atom. Finally, we note that the adhesive energy calculations described above are based on coherent interfaces, whereas the true density of the $\{111\} \mathrm{Cu}$ layers is about $36 \%$ greater than that of the interface oxygen layer:

$$
\rho_{m c} \equiv \frac{\rho_{\mathrm{Cu}}}{\rho_{\mathrm{O}}}=\left(\frac{a_{\mathrm{MgO}}}{a_{\mathrm{Cu}}}\right)^{2} \approx\left(\frac{7}{6}\right)^{2} \approx 1.36
$$

The interface bonding strength per $\mathrm{Cu}$ atom is somewhat reduced at the relative density $\rho_{m c}$ compared to the coherent case (for which $\rho_{m c}=1$ ). The simplest generalization of our treatment is to consider a density dependent prefactor $\varepsilon=\varepsilon\left(\rho_{m c}\right)$. We have performed some calculations, details of which will be described elsewhere, for periodic but incoherent interfaces with $\rho_{m c}=1.33$ and $\rho_{m c}=1.5$. The qualitative result is that if we consider the coherent $\{222\} \mathrm{MgO} / \mathrm{Cu}$ interface as the reference, and then densify the $\mathrm{Cu}$ layers, the binding of the additional $\mathrm{Cu}$ atoms to $\mathrm{O}$ is much less than the binding of the atoms in the coherent interface. Thus

$$
\left.\frac{d(\rho \varepsilon)}{d \rho}\right|_{\rho>1<<\varepsilon}
$$

In our numerical calculations we take $\varepsilon(1)=2.76 \mathrm{eV}$, and $\varepsilon(1.36)=2.0 \mathrm{eV}$, which corresponds approximately to $\frac{d(\rho \varepsilon)}{d \rho}=0$. 


\section{STRUCTURE SIMULATIONS FOR $\{222\} \mathrm{MgO} / \mathrm{Cu}$}

With these preliminaries regarding $E_{i}$ completed, we can now outline our molecular dynamics calculations for the $\{222\} \mathrm{MgO} / \mathrm{Cu}$ polar interface. As mentioned above, the $\mathrm{MgO}$ coordinates are frozen in their perfect-crystal configuration. The Cu EAM potential proposed by Johnson (1988), a computationally convenient analytical form, was employed

for $E_{m}$. We treat a computational unit cell with $7 \mathrm{Cu}$ and $6 \mathrm{O}$ atoms per side $(49 \mathrm{Cu}$ and 36 $\mathrm{O}$ atoms per layer) and $12 \mathrm{Cu}$ layers; as a control, we've performed another calculation with everything identical, except that the $\mathrm{Cu}$ interface layer has 6 atoms on a side and is therefore coherent with the oxygen. Larger computational cells will be used in future work. The atoms were started in perfect crystal positions and relaxed by a local optimization method, which is appropriate since no barriers exist to forming a dislocation network in this geometry (Gilmore 1989).

Figure 1 shows the relaxed atomic positions in the $\mathrm{Cu}$ interface layer projected onto a plane parallel to the interface (filled circles); the less densely packed $\mathrm{O}$ interface layer is represented by open circles. The simulated interface structure (which corresponds to $\mathrm{T}=0$ K) may be seen to exhibit an interfacial dislocation network of trigonal symmetry, consisting of dissociated partial dislocations lying along $(1 / 2)<110>$ directions with a $(1 / 6)<112>$ Burgers vector. Each unit cell of the trigonal network consists of two triangles, one of which corresponds to fcc and the other to hcp stacking at the interface. The other geometrically possible interfacial structure is an hexagonal network of perfect dislocations lying along $\langle 112\rangle$ directions with $(1 / 2)<110\rangle$ Burgers vectors. Incidentally, analogous dislocation networks were observed in simulations by Vellinga (1996) based on 
ad hoc interatomic potentials designed to have the correct periodicity of $\{222\} \mathrm{MgO} / \mathrm{Cu}$ interfaces, but otherwise not based on a detailed model of the bonding. Our calculations also show distortion of the layers normal to the interface, with interface $\mathrm{Cu}$ atoms near the on-top configuration displaced up to $0.2 \AA$ away from the mean layer position, $z \approx 1.3 \AA$. These distortions propagate out to the third layer, but essentially disappear by the fourth layer.

In addition to the simulations for misfit dislocations at the interface (in which the parameter $\varepsilon(1.36)=2.0 \mathrm{eV}$ was utilized), simulations were also perfomed (based on $\varepsilon(1)=2.76 \mathrm{eV}$ ) for a network with a single layer standoff. The network without standoff, however, is predicted to be energetically favored.

In principle, the trigonal and hexagonal networks are distinguishable by HREM (Vellinga 1996, Vellinga and De Hosson 1996) or Z-contrast STEM (Seidman et al. 1997, Shashkov 1997) but it has proven difficult, so far, to make a definitive identification on the basis of experiment alone. Recently Z-contrast microscopy was performed at Oak Ridge National Laboratory, in cooperation with M. Chisholm, employing their dedicated $300 \mathrm{kV}$ scanning transmission electron microscope (STEM) (Seidman et al. 1997, Shashkov 1997) on a $\{222\} \mathrm{MgO} / \mathrm{Cu}$ interface with segregated $\mathrm{Ag}$ solute atoms (Shashkov and Seidman 1995, Shashkov 1997, Shashkov et al. 1997). The present simulation approach is being generalized to enable treatment of segregated Ag solutes, and comparison with these experiments. Furthermore, comparisons of the present simulations will be made with Zcontrast STEM observations in progress on a pristine $\{222\} \mathrm{MgO} / \mathrm{Cu}$ interface, as well as interfaces with segregated $\mathrm{Ag}$ atoms. 


\section{INTERFACE ELECTRONIC STRUCTURE}

In spite of the large misfit at the $\{222\} \mathrm{MgO} / \mathrm{Cu}$ interface, coherent patches cover much of the interface area, and therefore electronic structure calculations based on the coherent interface approximation are expected to yield useful insights into the true interface. Layerintegrated density of electronic states calculations were performed in the vicinity of the oxygen-terminated $\{222\} \mathrm{MgO} / \mathrm{Cu}$ interface within the coherent interface approximation (Figure 2). These calculations utilized a multilayer geometry with $5 \mathrm{Mg}$ layers, $6 \mathrm{O}$ layers and $6 \mathrm{Cu}$ layers per unit cell; the unit cell thus has two identical oxygen-terminated $\mathrm{MgO} / \mathrm{Cu}$ interfaces. The local density of states is integrated over slabs centered on each of the layers; the $\mathrm{Cu}$ and $\mathrm{O}$ layers at the interface were combined, however. The energy zero corresponds to the Fermi energy. Except for the layers adjoining the interface itself, the layers on both sides of the interface show relatively bulklike behavior. The peak at $-17 \mathrm{eV}$ on the $\mathrm{MgO}$ side corresponds to the $\mathrm{O} 2 \mathrm{~s}$ band. This peak is slightly shifted to higher energy at the interface, which reflects an electrostatic potential shift. We note that the $\mathrm{Cu}$ dband edge appears a few tenths of an $\mathrm{eV}$ higher than in bulk $\mathrm{Cu}$ calculations, as a result of the expansion of $\mathrm{Cu}$ to achieve coherence with $\mathrm{MgO}$. A significant feature at the interface is a peak in the density of states at about $0.5 \mathrm{eV}$ below the Fermi energy, within the $\mathrm{MgO}$

energy gap. This peak appears to represent an interface state (or "metal-induced gap state" (Finnis 1996)) that decays exponentially into the $\mathrm{MgO}$. It is most likely a hybrid of $\mathrm{Cu}$ $(4 s)$ and $\mathrm{O}(2 \mathrm{p})$ states. The perturbation of the electronic states at the interface may be observable by EELS (Muller et al. 1996), and such experiments are in progress.

\section{SUMMARY}


Atomistic simulations are performed for a polar ceramic/metal interface with misfit included. Polar interfaces as well as the effect of misfit have been largely neglected in previous atomistic simulations. LDFT calculations (Benedek et al. 1996) suggest that the O-terminated $\{222\}$ interface, the subject of this work, is the $\mathrm{MgO} / \mathrm{Cu}$ interface with highest adhesive energy, and this is the only interface configuration observed experimentally for $\mathrm{MgO}$ precipitates in $\mathrm{Cu}$ (Jang et al. 1993, Seidman et al. 1997, Shashkov et al. 1997). We have developed a short-range interatomic potential model for the interface interaction $E_{i}$, guided by ab initio LDFT calculations. Based on the model, we predict a misfit dislocation network of trigonal symmetry, with no standoff dislocations in the pristine interface. Our electronic structure calculations exhibit an interface state at a binding energy of $0.5 \mathrm{eV}$.

\section{ACKNOWLEDGMENTS}

R. Benedek and D. N. Seidman were supported at Northwestern University by the U. S. Department of Energy, Office of Basic Energy Sciences, under grant DEFG02-

96ER45597. L. H. Yang was supported at Lawrence Livermore National Laboratory by the U. S. Department of Energy under contract No. W-7405-ENG-48. Most of the numerical calculations were performed at the National Energy Research Supercomputer Center.

\section{REFERENCES}

Benedek, R., Minkoff, M. and Yang, L. H. (1996), Phys. Rev. B 54, 7697. 
Duffy, D. M., Harding, J. H., and Stoneham, A. M. (1995), Acta Metall. Mater. 43, 1559.

Finnis, M. W. (1992), Acta Metall. Mater. 40, S25.

Finnis, M. W. (1996), J. Phys.: Condens. Matter 8, 5811.

Gilmore, C. M. (1989), Phys. Rev. B 40, 6402.

Jang, H., Seidman, D. N., and Merkle, K. L., (1993), Interface Science 1, 61.

Johnson, R. A. (1988), Phys. Rev. B 37, 3924.

Muller, D. A., Subramanian, S., Batson, P. E., Sass, S. L., and Silcox, J. (1995), Phys.

Rev. Lett. 75, 4744.

Purton, J., Parker, S. C., and Bullett, D. W. (1996), preprint.

Seidman, D. N., Shashkov, D. A., Benedek, R., and Chisholm, M. F. (1997), this

proceedings.

Shashkov, D. A. (1997) Ph.D. thesis, Northwestern University.

Shashkov, D. A., Chan, D. K., Benedek, R., and Seidman, D. N. (1997) to appear in

Trans. Japanese Institute Metals.

Shashkov, D. A. and Seidman, D. N. (1995), Phys. Rev. Lett. 75, 268.

Smith, J. R., Hong,T., and Srolovitz, D. J. (1994), Phys. Rev. Lett. 72, 4021.

Vitek, V., Gutekunst, G., Mayer, J., and Rühle, M. (1995), Phil. Mag. A 71, 1219.

Vellinga, W. P. (1996 ), Ph. D. Dissertation, University of Groningen.

Vellinga, W. P., and De Hosson, J. Th. M. (1996), Mat. Sci. Forum 207-209, 351. 


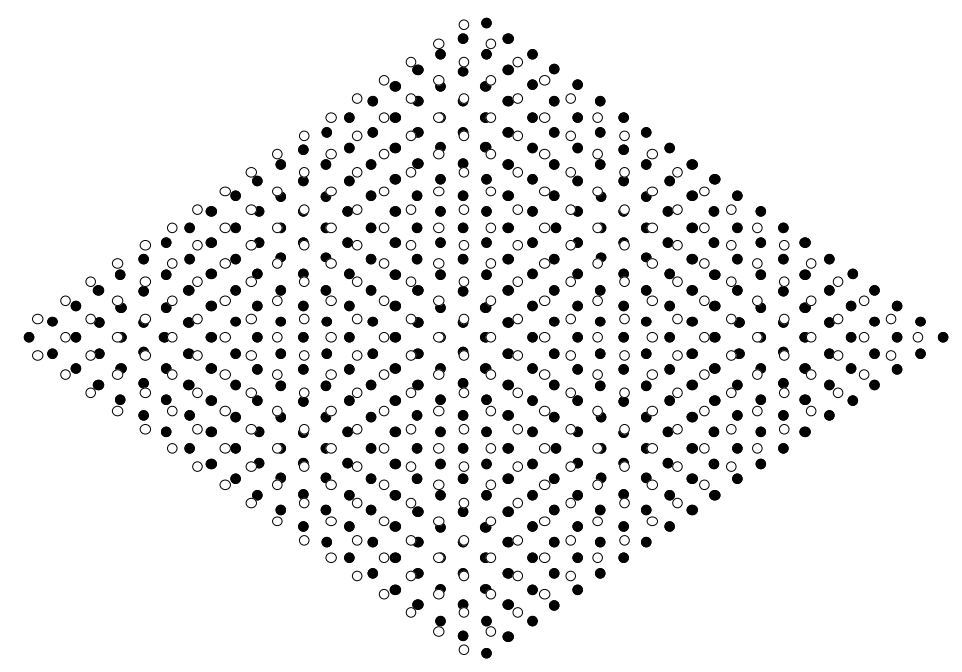

Fig. 1. Relaxed interface layer atomic positions projected onto the plane of the interface for $\mathrm{Cu}$ (filled circles) and $\mathrm{O}$ (open circles) calculated for oxygen-terminated $\{222\} \mathrm{MgO} / \mathrm{Cu}$ interface. 


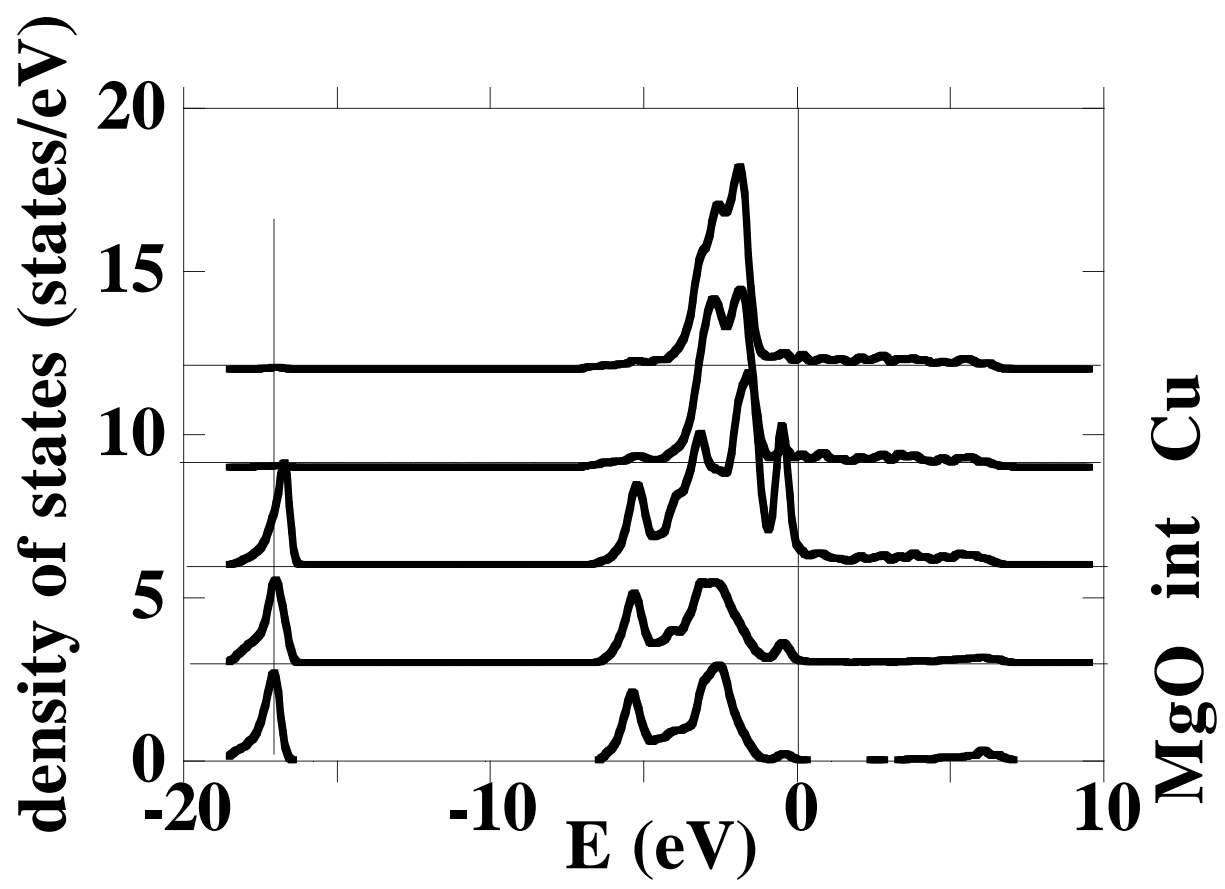

Fig. 2. Electronic density of states in vicinity of $\{222\} \mathrm{MgO} / \mathrm{Cu}$ interface. The separate curves are obtained by integrating over slabs parallel to the interface. The curve labelled int superposes the $\mathrm{Cu}$ and the $\mathrm{O}$ layer at the interface. Also shown are results for the two adjacent layers on either side of the interface. $\mathrm{Mg}$ and $\mathrm{O}$ sublayers are combined, except at the interface. 


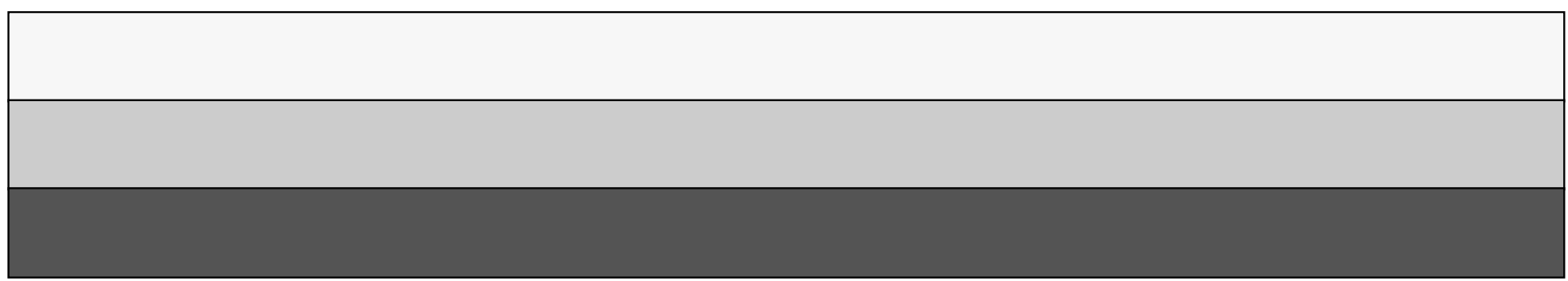

